\title{
The role of neoadjuvant chemotherapy in LABC - A multicentric study
}

\author{
Rupa M. Mascarenhas ${ }^{1}$, Manish Kumar ${ }^{2}$, Elroy Saldanha ${ }^{3}$, Sheldon Mathias ${ }^{4 *}$, Rachan Shetty ${ }^{5}$ \\ ${ }^{1,5}$ Assistant Professor, ${ }^{2}$ Senior Resident, ${ }^{3}$ Resident, ${ }^{4}$ Associate Professor, ${ }^{\mathbf{1 - 4}}$ Dept. of General Surgery, ${ }^{\mathbf{5}}$ Dept. of Oncology, ${ }^{\mathbf{1 , 5}} \mathrm{AJ}$ Institute of \\ Medical Sciences \& Research Centre, Mangalore, Karnataka, ${ }^{2,4}$ Father Muller Medical College Hospital, Mangalore, Karnataka, ${ }^{3}$ Sri \\ Aurobindo Institute of Medical Sciences, Indore, Madhya Pradesh, India
}

*Corresponding Author: Sheldon Mathias

Email: sheldon98@gmail.com

\begin{abstract}
Introduction: LABC accounts for 10-20\% in the West, while in India, it accounts for $30-60 \%$ of all cases. LABC encompasses a wide spectrum of malignant breast tumors with varying presentation and poses a significant therapeutic challenge. Locally advanced breast cancer presents with a difficult management problem. It remains a challenge to achieve local and distant control of locally advanced breast cancer. Locally advanced breast cancer presents with a difficult management problem. It remains a challenge to achieve local and distant control of locally advanced breast cancer neoadjuvant chemotherapy in LABC.

Materials and Methods: A prospective, non-interventional study was done on multiple centres in the district of Dakshina Kannada on 100 adult patients admitted in who either presented with histological confirmed locally advanced breast cancer were be included in the study as per the following criteria between June 2014 to May 2019. After this preoperative work up all patients. We used AC regimen in 120 patients. The Response to chemotherapy was studied in all patients who received neo adjuvant chemotherapy according to the Response Evaluation Criteria in Solid Tumors published in February 2000 by European Organization for Research and Treatment of Cancer was used to evaluate the tumor response The patients are followed up for a period of minimum of 3 years and maximum 2 years and at regular intervals during each cycle of chemotherapy. Clinical examination in details about the tumor characteristics and change in size of tumor with status of axillary LN had been entered in the database. After completion of NACT all patients assess for operability and underwent modified radical mastectomy. The surgical specimens removed from all the patients are sent for histopathology examination in the pathology section and are studied for Pathological response and Histological grade.

Observations and Results: In the present study, the age of the cases in the study ranged from 22 years to 79 years, 56.72 years SD + 15 years; the most common age group was $40-55$ years $48 \%$ of the entire study population. Less than 25 years we had 2 cases between $25-35$ we had 12 cases all had a family history of cancer. 78\% used OPS for contraception. Clinical and pathological complete response was seen more commonly in Her2 subtype cases. NACT could downstage the disease so as to make the inoperable tumor to operable one and possible to resect locally advanced disease.
\end{abstract}

Keywords: Clinical, Neoadjuvant, Pathological, Locally advanced breast cancer.

\section{Introduction}

LABC (Locally Advanced Breast Cancer) accounts for 10$20 \%$ of all breast cancers in the West, while in India, it accounts for $30-60 \%$ of all cases. ${ }^{1}$ LABC comprises a wide spectrum of malignant breast tumors with varying clinical presentation and pose a significant challenge to treat as it is very difficult to achieve a local and distant control of locally advanced breast cancer ${ }^{2}$ with the newer concepts in the management of breast cancer the treatment of breast cancers, the goal of management of in the present day in treating breast cancers is achieving a good cosmetic results along with giving a disease free period to the individual. In view of this we did a multicentric study to evaluate the role of neoadjuvant chemotherapy in localized advanced breast cancer management.

\section{Materials and Methods}

A prospective, non-interventional study was done on multiple centres in the district of Dakshina Kannada on 100 adult patients admitted in who presented with histologically confirmed locally advanced breast cancer between June 2014 to May 2019. As per the defined criteria we had 120 patients who consented to participate in the study by their free will. The regimen that was used in all the patients was AC regimen. The response to chemotherapy was studied in all patients.
Those patients who received neo adjuvant chemotherapy were evaluated for the tumor response according to the Response Evaluation Criteria in Solid Tumors published in February 2000 by European Organization for Research and Treatment of Cancer.

The patients were followed up for a period of minimum of 2 years and maximum 3 years and at regular intervals and during each cycle of chemotherapy.

The clinical examination details that included the tumor characteristics with respect to the change in size of tumor, status of axillary all were collected after completion of NACT all patients assessed for operability and those who were found to be operable underwent modified radical mastectomy. The surgical specimens removed from all the patients are sent for histopathology examination and the pathology section and are studied for Pathological response and Histological grade.

The collected data was and transferred to a master chart for analysis and analysis was done by SPSS software 23.0 version.

\section{Observations and Results}

In the present study, the age of the cases in the study ranged from 22 years to 79 years, 56.72 years $S D \pm 15$ years; the most common age group was $40-55$ years $48 \%$ of the entire study population. Less than 25 years we had 2 cases 
.between 25-35 we had 12 cases all had a family history of cancer $.78 \%$ used oral contraceptive pills for contraception. Most common adverse effect is alopecia 60 cases, mucositis 24 cases nausea in $35 \%$ diarrhea $12 \%$ cases. ER/PR receptor was Positive in 72 equivocal in 3 cases and negative in 25 cases. HER2n receptor was positive in 10 cases. We had 3 cases of triple negative disease. In the present study $92 \%$ had histology of infiltrating ductal carcinoma, and $8 \%$ had histology of infiltrating lobular carcinoma.

\section{Table 1}

\begin{tabular}{|c|c|}
\hline Clinical Response & Frequency \\
\hline cCR & 28 \\
\hline cPD & 2 \\
\hline cPR & 60 \\
\hline cSD & 10 \\
\hline Total & 100 \\
\hline Pathological Response & Frequency \\
\hline Partial response & 83 \\
\hline complete response & 17 \\
\hline Total & 100 \\
\hline
\end{tabular}

\section{Discussion}

Breast cancer is the most common site-specific cancer in women and is the leading cause of death from cancer for women Recent guidelines from the U.S. National Comprehensive Cancer Network describe LABC as AJCC stage III breast cancer; the definition includes breast cancer that fulfils any of the following criteria in the absence of distant metastasis: ${ }^{4}$ Tumours more than $5 \mathrm{~cm}$ in size with regional lymphadenopathy (N1-3). Tumours of any size with direct extension to the chest wall or skin, or both (including ulcer or satellite nodules), regardless of regional lymphadenopathy. Presence of regional lymphadenopathy (clinically fixed or matted axillary lymph nodes, or any of infraclavicular, supraclavicular, or internal mammary lymphadenopathy) regardless of tumour stage. Locally advanced breast cancer presents with a difficult management problem. It remains a challenge to achieve local and distant control of locally advanced breast cancer. ${ }^{4}$ Over the last decade preoperative/ neo-adjuvant chemotherapy has emerged as the standard of care for these patients. ${ }^{5}$ Successful reduction in the size of the tumor is associated with increased rate of operability i.e. modified radical mastectomy or Breast Conserving Therapy (BCT). Also set of cases with stage IIB and stage IIIA considered operable have been treated with surgery followed by adjuvant chemotherapy and/or hormonal modality. Guilherme Freire Angotti Carrara et al showed a pathologic complete response was observed in $27.6 \%$ of the tumors ${ }^{4}$ Tapesh Bhattacharyya et al in 2015 studied the factors influencing response to NACT, Complete response was seen in $27(18.2 \%)$ patients and $92(62.2 \%)$ patients showed partial response. Pathological complete response was seen in $24(16.2 \%)$ patients. ${ }^{5}$ Sataloff (1995) on 36 patients who underwent neoadjuvant chemotherapy for LABC had a ccr\% and pcr\% of $33 \%$ and $39 \%$ respectively. ${ }^{6}$ Cocconi
(1990) on 48 patients who underwent neoadjuvant chemotherapy for LABC had a ccr\% and pcr\% of $8 \%$ and $14 \%$ respectively. ${ }^{7}$ Iqbal et al complete response was achieved in $24(44.4 \%)$ patients, $14(25.9 \%)$ of the patients had partial response and $16(29.6 \%)$ progressed clinically. Average $^{8}$ all the above studies also showed that in histology $\mathrm{f}$ infiltrating ductal carcinoma was the commonest, which is similar to our study.

\section{Conclusions}

Clinical and pathological complete response was seen more commonly in Her2 subtype cases. NACT could downstage the disease so as to make the inoperable tumor to operable one and possible to resect locally advanced disease.

\section{Conflict of Interest: None.}

\section{References}

1. Singletary SE, Allred C, Ashley P, Bassett LW, Berry D, Bland KI, Borgen PI, Clark G, Edge SB, Hayes DF, Hughes LL. Revision of the American Joint Committee on Cancer staging system for breast cancer. J Clin Oncol 2002;20(17):3628-36.

2. Giordano SH. Update on locally advanced breast cancer. Oncologist 2003;8(6):521-30.

3. National Comprehensive Cancer Network (nccn) NCCN Clinical Practice Guidelines in Oncology: Breast Cancer. Fort Washington PA: NCCN; 2015. Ver. 2.2015. [Current version available online at:http://www.nccn.org/professionals/physician_gls/pdf/breast. pdf (free registration required); cited 12 April 2012.

4. Carrara GF, Scapulatempo-Neto C, Abrahão-Machado LF, Brentani MM, Nunes JS, Folgueira MA, Vieira RA. Breastconserving surgery in locally advanced breast cancer submitted to neoadjuvant chemotherapy. Safety and effectiveness based on ipsilateral breast tumor recurrence and long-term follow-up. Clinics 2017;72(3):134-42.

5. Bhattacharyya T, Sharma SC, Yadav BS, Singh R, Singh G. Outcome of neoadjuvant chemotherapy in locally advanced breast cancer: A tertiary care centre experience. Indian J Med Paediatr Oncol: J Indian Soc Medical \& Paediatr Oncol 2014;35(3):215.

6. Sataloff DM, Mason BA, Prestipino AJ, Seinige UL, Lieber patholoogical response to induction chemotherapy in LABC: A determinant of outcome. J Am Coll Sug 1995;180:297-306.

7. Cocconi G, Di Blasio B, Boni C, Bisagni G, Rondini E, Bella MA, Leonardi F, Savoldi L, Vallisneri C, Camisa R, Bruzzi P. Primary chemotherapy in operable breast carcinoma comparing CMF (cyclophosphamide, methotrexate, 5fluorouracil) with an anthracycline-containing regimen: Shortterm responses translated into long-term outcomes. Ann Oncol 2005;16(9):1469-76.

8. Iqbal J, Shafi AA, Alharthi BN. Neoadjuvant chemotherapy in locally advanced breast cancer. J Coll Phys Surg Pak 2014;24(11):845-8.

How to cite this article: Mascarenhas RM, Kumar M, Saldanha E, Mathias S, Shetty R. The role of neoadjuvant chemotherapy in LABC - A multicentric study. Indian $J$ Anat Surg Head Neck Brain 2019;5(2):59-60. 